Journal of Applied Fluid Mechanics, Vol. 6, No. 1, pp. 81-86, 2013.

Available online at www.jafmonline.net, ISSN 1735-3572, EISSN 1735-3645.

DOI: $10.36884 / \mathrm{jafm} .6 .01 .19495$

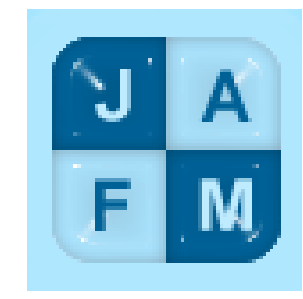

\title{
Breakup of Droplets in Micro and Nanofluidic T-Junctions
}

\author{
A. Bedram ${ }^{1 \dagger}$ and A. Moosavi ${ }^{2}$ \\ ${ }^{1,2}$ Mech. Eng. Dep., Sharif University of Technology, P. O. Box: 11365-9567, Tehran, Iran \\ $\dagger$ Corresponding Author Email: bedram@iran.ir
}

(Received November 17, 2010; accepted August 3, 2011)

\begin{abstract}
We employ numerical simulations to investigate the breakup of droplets in micro- and nanoscale $\mathrm{T}$ junctions, which are used to produce small droplets from a large droplet. For this purpose a Volume f Fluid (VOF) based method is used and for verifying the reliability of the numerical outcomes, the results are compared with the available experimental and analytical results. Our results reveal that breakup time and breakup length of the droplets play important roles in handling these systems optimally. Our results also indicate that for nanoscale Tjunctions by increasing the capillary number the performance increases while for the micro-scale systems there is a specific capillary number for which the system is in its optimum condition.
\end{abstract}

Keywords: Micro- and nanoscale, T-junctions, Droplet breakup, VOF

\section{INTRODUCTION}

In many industrial applications, it is necessary to mix, analyze and handle minute quantities of liquids (Tice et al. 2003; Burns et al. 1998; Cristini and Tan 2004; Prakash and Gershenfeld 2007; Sybulski and Garstevki 2010). Droplet based microfluidic systems have been shown to be a promising tool for this purpose. These systems allow one to conveniently handle small amounts of liquids for various processes.

Producing large number of droplets with different sizes in a short time is a prerequisite to use these systems efficiently. Since this problem also occurs in other applications (for example production of emulsions in pharmacy and chemical industries), a variety of methods for this purpose has been proposed and are available (Sugiura et al. 2001; Ganan 1998; Thorsen et al. 2001; Anna et al. 2003; Link et al. 2004). However, using a series of $T$ junctions and breaking up a large droplet to a large number of small droplets has been shown to be an efficient method for micro- and nanofluidic applications (Link et al. 2004). Thus, breakup of droplet in T junctions have been extensively studied both experimentally (Link et al. 2004) and numerically (Urbant et al. 2008; Gupta et al. 2009; Bedram and Moosavi 2010, 2011).

In the present study, a symmetric T-junction at both the micro- and nanoscale has been considered (for generation of large number of droplets) and studied numerically. Two important parameters, namely, the droplet breakup time and the droplet breakup length have been investigated. These two parameters that were first introduced by Bedram and Moosavi (2010), play important role in decreasing the cost and increasing the performance of the process of droplets generation in T-junctions. Also, in the present study, we try to explore the possible difference between the operation of these systems at the micro- and nanoscales. In order to check the accuracy and reliability of the results, the results of the study have been compared with the available analytical results (Leshansky and Pismen 2009; Bretherton 1961).

\section{Numerical Algorithm}

In order to study the system, two non-volatile, incompressible and immiscible fluids were considered and the continuity and Navier-Stokes equations with the associated boundary conditions were solved for the system using a Volume of Fluid (VOF) based method. The problem can be modeled by development of an already available code (Lafaurie et al. 1994; Scardovelli and Zaleski 1999). In more detail, the method can be described as follows: The continuity condition for the systems can be simply stated as

$\nabla \cdot \mathrm{u}=0$ 
where $\mathbf{u}$ is the velocity vector. The momentum equation can be cast into the following mathematical form

$$
\rho\left(\partial_{t} \mathrm{u}+\mathrm{u} \cdot \nabla \mathrm{u}\right)=-\nabla p+2 \nabla \cdot \mu \mathrm{D}+2 \gamma \kappa \delta_{S} \mathrm{n}
$$

where $\rho$ and $\mu$ represent the density and viscosity, respectively and are calculated as

$$
\begin{aligned}
& \rho=\rho_{c} \varphi+\rho_{d}(1-\varphi) \\
& \mu=\mu_{c} \varphi+\mu_{d}(1-\varphi)
\end{aligned}
$$

with $\phi$ as the volume fraction of the continuous phase. The subscripts $c$ and $d$ stand for the continuous and dispersed phases (droplet), respectively. $\phi$ in each cell is a value between 0 to 1 and is derived from

$$
\frac{\partial \varphi}{\partial t}+\mathrm{u} \cdot \nabla \varphi=0
$$

In Eq. (2) $\mathbf{D}$ is the rate of stress tensor and can be calculated from

$D_{i j}=\frac{1}{2}\left(\frac{\partial u_{j}}{\partial x_{i}}+\frac{\partial u_{i}}{\partial x_{j}}\right)$

with $x_{i}$ as the $i$-component of the position vector. $\gamma$ is the surface tension at the interface between two fluids, $\kappa$ represents the mean curvature of the interface, $\delta_{S}$ is a delta function on the surface $S$ and $\mathbf{n}$ is the interface unit normal vector (Karniadakis $e t$ al. 2004).

The method solves the equations of momentum and continuity simultaneously for each of the fluids. The boundary is situated on the regions where $\phi$ is equal to 0.5 . A piecewise linear interface reconstruction method is used to construct the boundary. For the surface tension a continuum surface force (CSF) model is also used (Renardy 2007; Brackbill et al. 1992).

The numerical procedure is checked to make sure that the results are not depending on the grid size and the time step size. A QUICK routine is used for differencing the momentum equation and a SIMPLEC algorithm has been employed to couple the pressure and velocity. The convergence criterion is considered to the residuals be less than 0.0005 .

If Knudsen number is a value between 0.01 and 0.1 one can still use the continuity and Navier-Sokes equations but no slip condition is not valid anymore (Karniadakis et al. 2004). Thus for nanoscale cases, we apply the slip condition at the solid-liquid interface.

The capillary number is defined as

$$
C a=\frac{\mu_{c} U_{c}}{\gamma}
$$

where $\mu_{c}$ represents the viscosity of the continuous fluid, $\gamma$ stands for the surface tension and $U_{c}$ is the average velocity of the continuous fluid in one of the branches that for our problem, since the inlet velocity is a known value the velocity of the droplet, it is equal to one half of the input velocity. In general, the average velocity of the continuous phase in branches can be calculated via,

$U_{c}=\frac{\int_{A} u d A}{A}$

Where $A$ represents the cross section area of the branch. The calculation is done when the cross section is fully occupied by the continuous phase.

Before considering the major task, the accuracy and reliability of the numerical algorithm is checked by two benchmarks. First, we calculate numerically the critical length $^{1}$ as a function of the capillary number for breakup of the droplets in $\mathrm{T}$-junctions and compare the results with the analytical solution of Leshansky and Pismen (2009) that shown in Fig. 1. In the second benchmark we numerically calculate the velocity of a slender droplet in a circular tube and compare the results with the analytical solution of Bretherton (1961) that shown in Fig. 2. Very close agreement were found for two cases considered as depicted in Figs. 1 and 2.

\section{Results}

One way of producing large number of droplets is transferring the droplets through several $\mathrm{T}$ shaped junctions as depicted in Fig. 3. By decreasing the distance between the droplets, more droplets can enter the T-junction in a period of time and consequently this will increase the rate of droplet generation. The minimum distance between the droplets can be obtained from

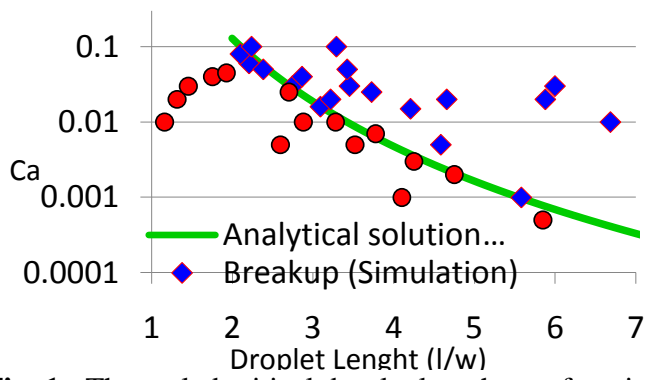

Fig. 1. The scaled critical droplet length as a function of capillary number. $\mathrm{w}$ is the channel width before in inlet channel.

\footnotetext{
${ }^{1}$ In a T-junction if the length of droplet was less than a specific value (Critical length) the droplet don't breakup but enter into one of the branches.
} 


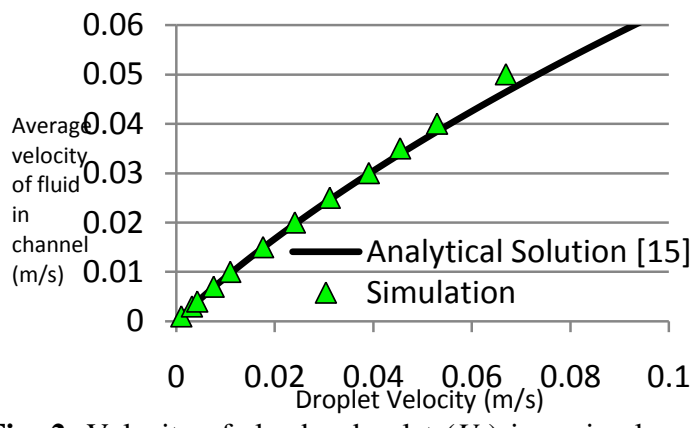

Fig. 2. Velocity of slender droplet $\left(U_{d}\right)$ in a circular tube as function of average velocity of continuous phase in the inlet channel.

$X_{\min }=U_{d} \times t_{\text {breakup }}$

Where $X$ is the distance between the droplets and $U_{d}$ expresses the velocity of the droplets. $t_{\text {breakup }}$ is the breakup time of the droplets in $\mathrm{T}$ junction (see Fig. 4). $t_{\text {breakup }}$ is the time of the whole procedure from Fig. 4-a to Fig. 4-b that was first introduced by Bedram and Moosavi (2010).

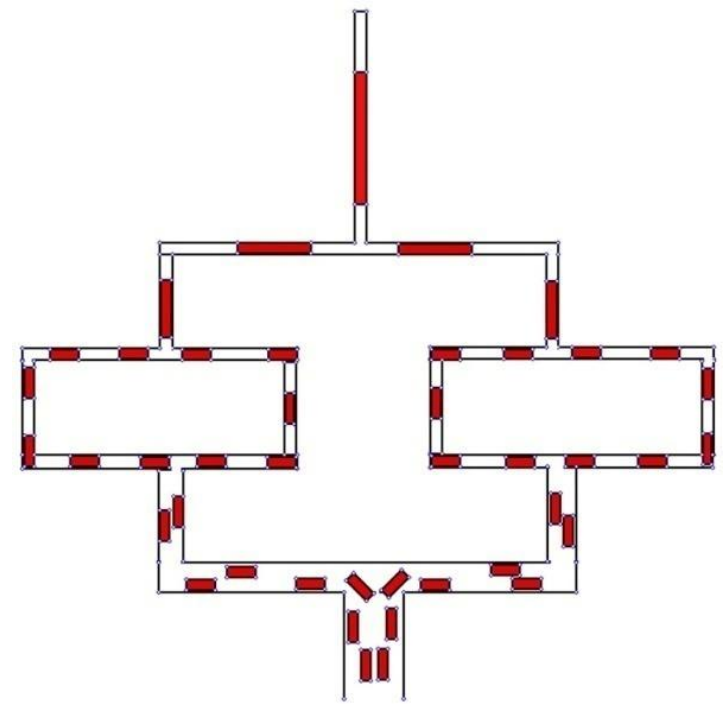

Fig. 3. A schematic figure of production of large number of droplets in consecutive T-junctions.

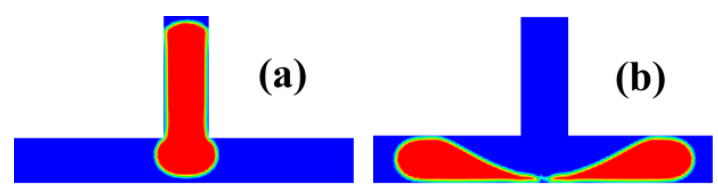

Fig. 4. The breakup of droplet in a T-junction. The breakup time is the time of the whole procedure between figure (a) and figure (b).

In the $\mathrm{T}$ junction system used for the breakup process, because of the size and the flow conditions considered, the effect of inertia and gravity on the breakup processes can be neglected. As Garstecki et al. (2006) illustrated, there are three important forces involved in the process, namely, interfacial (surface tension), shear (viscous) and hydrodynamics resistance (pressure) forces. Surface tension forces are stabilizing (against the breakup of the droplet) and produce a net force on the tip oriented upstream. Shear stress forces between the wall and the droplet are also stabilizing but shear stress force between the two fluids and pressure forces are destabilizing (responsible for breakup) and produce a net force downstream. The interfacial forces are proportional to the surface tension while the shear stress forces between the two fluids and the pressure forces are proportional to the velocity of the continuous phase (Garstecki et al. 2006). The shear stress between the wall and the droplet is proportional to the velocity of the droplet and as a result proportional to the velocity of the continuous phase (Baroud et al. 2010). Therefore, the breakup process can be discussed in terms of the capillary number.

Figures 5 and 6 show the breakup time as a function of the capillary number for the micro- and nanoscale cases, respectively. In microscale case for $\mathrm{Ca}>0.05$ the profile of the droplet because of increasing the ratio of dynamical forces to the viscous and surface tension forces experiences a deformation. Therefore, it is not possible to model these limits but in nanoscale cases, since the surface tension is stronger than the microscale case, the droplet profile is such that it still can be modeled.

As depicted in Fig. 5 the breakup time for $C a>0.025$ is a constant value and for $C a<0.02$ it is proportional to $\mathrm{Ca}^{-0.13}$. This sudden change is because of this concept that for $\mathrm{Ca}<0.02$ the droplet and the wall are in contact with each other (Fig. 7-a) and as a result the shear stress forces between the wall and the droplet, which are destabilizing, participate in the process of the breakup. By decreasing the capillary number the contact area between the droplet and the wall increases and this boosts the destabilizing forces. As a result by decreasing the capillary number the breakup time increases.

For $\mathrm{Ca}>0.025$ the droplet does not touch the top surface as depicted in Fig. 7-b. Thus, only fluid-fluid shear stress forces take part in the process. Because of this canceling the role of wall-fluid shear stress forces in the process, there is a discontinuity in the profile of the breakup time. However, in the nano case there is no discontinuity in the profile of the breakup time. 


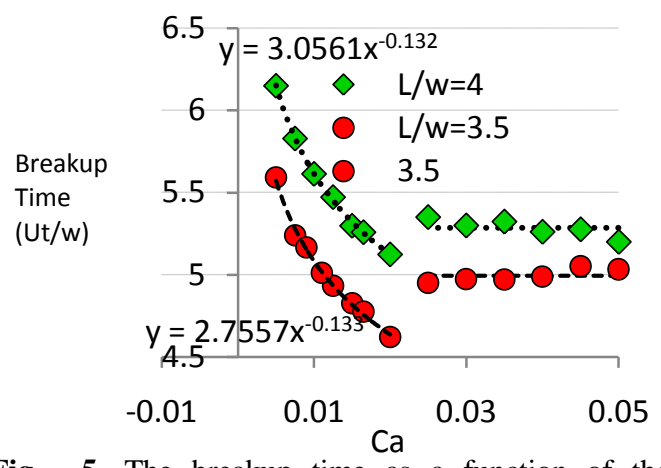

Fig. 5. The breakup time as a function of the capillary number for microscale case (Bedram and Moosavi 2010). U and w are the channel width and the average velocity of the fluid carrying the droplets before the T-junction, respectively.

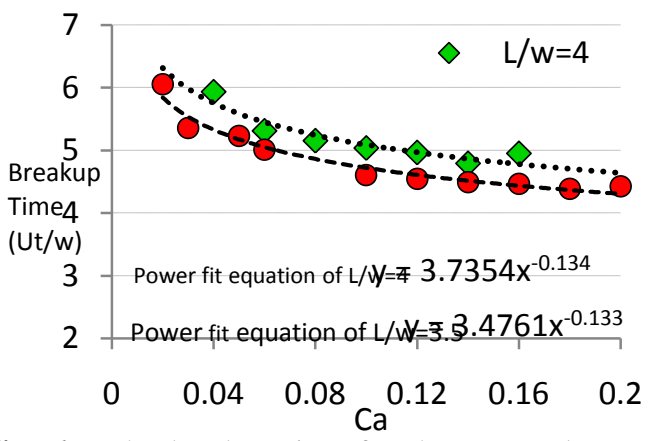

Fig. 6. The breakup time for the nanoscale case. Similar to Fig. 5, U and $w$ are the channel width and the average velocity of the fluid carrying the droplets before the T-junction, respectively.

For this case because of the slip condition on the walls, the droplets always are in contact with the walls. Therefore in the nanoscale, for increasing the production speed (in the system of Fig. 3) the capillary number should be increased as much as possible. But for micro scale there is a specific capillary number for which the rate of droplet generation in the system of Fig .3 will be maximal.

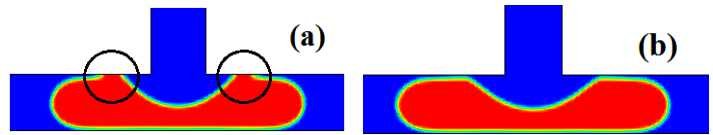

Fig. 7. Mechanism of breakup of droplets in micro Tshaped junctions for $\mathrm{Ca}=0.02$ (a) and $\mathrm{Ca}=0.025$ (b). $\mathrm{L} / \mathrm{w}=3.5$ for the cases.

Interestingly for nanoscale cases we found that that a while before the breakup, the profile of the top surface of the droplet becomes pricewise linear (Fig .8-a).

The parameter "droplet breakup length" has an essential role in reducing the production costs. As displayed in Fig. 3 after a T-junction there is an 90 degree elbow. By decreasing the distance between the elbow and the junction, smaller length of tube will be used and the cost of system will be reduced.
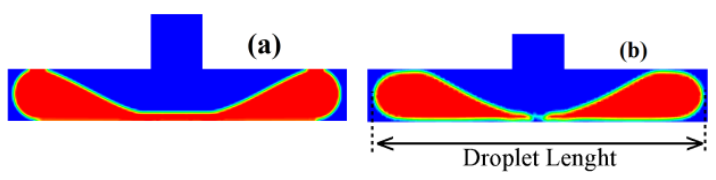

Fig. 8. a) Piecewise linear profile of the droplets during the breakup for the nanoscale case. b) Definition of length of droplet breakup.

The minimum distance between the elbow and the junction should be such that, a small gap between the droplet and the elbow should remain after breakup of the droplet. Therefore, it is necessary to calculate the droplet breakup length (Fig. 8-b). In Figs. 9 and 10 this parameter as a function of the capillary number for both the microscale and nanoscale cases are shown.

According to Fig. 9 the droplet breakup length linearly increase for $\mathrm{Ca}>0.025$ and it is of the form $\mathrm{Ca}^{-0.07}$ for $\mathrm{Ca}<0.02$. As already discussed, this feature stems from the contact between the droplet and the wall surfaces. Therefore, in the microscale case, the droplet breakup length has its minimum value in $\mathrm{Ca}=0.016$ and, as a result, the production cost will be minimum. However, in the nanoscale case (Fig. 10) there is no minimum because of the slip effect. Thus, the capillary number should be increased as much as possible.

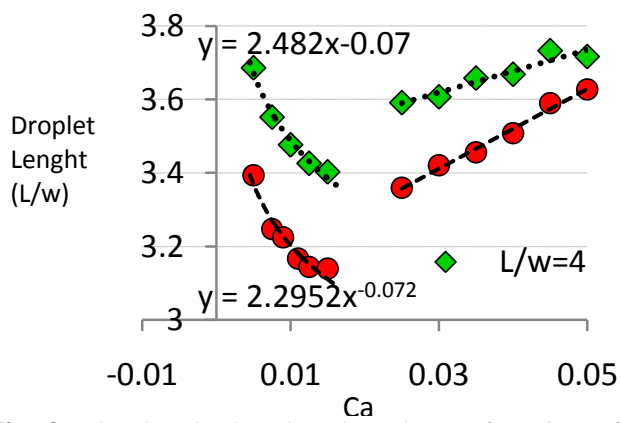

Fig. 9. The droplet breakup length as a function of the capillary number for the microscale case (Bedram and Moosavi 2010).

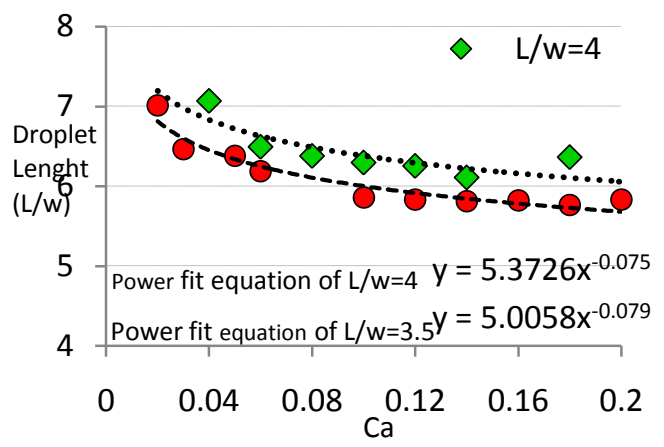

Fig. 10. The droplet breakup length as a function of the capillary number for the nanoscale case. 


\section{ConClusion}

A VOF numerical routine was utilized as a tool to find possible ways to reduce the costs and increase the production rate of droplet in $\mathrm{T}$ junction systems. A T-junction was considered and the effects of two important parameters, i.e., the "breakup time" and the "breakup length" were studied. It was observed that in order to increase the efficiency and reduce the costs of above system at the nanoscales, the capillary number should be increased while at the miroscale case, there is an optimum value for the capillary number $(\mathrm{Ca}=0.02)$. Also a qualitative difference between the profile of the micro- and nanodroplets during the breakup was also observed.

As has been explained in the text there are several factors that restrict the range of the applicability of the results. For larger values of the Knudsen number the fluids cannot be considered continuous and the continuity and Navier-Stokes equations are not valid anymore. Also, because of deformations, the results have been obtained for $\mathrm{Ca}<0.5$. The investigations have been done for $2 \mathrm{D}$ droplets but as outlined by Leshansky and Pismen (2009) there are many close agreements between the results of $2 \mathrm{D}$ and $3 \mathrm{D}$ (for instance, the location of boundary of breakup and non-breakup regions as a function of the initial extension of the droplets and the capillary number.), (Bretherton 1961). One important parameter that may affect the results is the viscosity ratio. All the investigations in the present study have been carried out for a viscosity ratio equal to 1.25 and further investigations should be done to study the effects of this ratio. Jullien et al. (2009) have shown that changing the viscosity ratio does not change the overall picture of the behavior of droplets in a $\mathrm{T}$ junction but the observed regimes occur for different values of the capillary numbers and the initial extension of the droplets.

\section{REFERENCES}

Anna S.L., N. Bontoux, and H.A. Stone (2003). Formation of dispersions using "flow focusing" in microchannels. Appl Phys Lett. 82, 364-366.

Baroud C. N., F. Gallaireb, R. Dangla (2010). Dynamics of microfluidic droplets. Lab on $a$ Chip. 10, 2032-2045.

Bedram A. and A. Moosavi, (2010). Numerical investigation of droplets breakup in microfluidic T-junction. International Conference on Physics Science and Technology ICPST. Hong Kong. Chin,. 162-166.

Bedram A. and A. Moosavi, (2011), Droplet breakup in an asymmetric microfluidic $\mathrm{T}$ junction. Eur. Phys. J. E. 34: 78
Brackbill J.U., D. B. Kothe and C. Zemach (1992). A continuum method for modeling surface tension. J. Comp. Phys. 100, 335-354.

Bretherton F.P. (1961). The motion of long bubbles in tubes. J. Fluid Mech. 10, 166-188.

Burns M.A., B. N. Johnson, S. N. Brahmasandra, K. Handique, J. R. Webster, M. Krishnan, T. S. Sammarco, P. M. Man, D. Jones, D. Heldsinger, C. H. Mastrangelo and D. T. Burke (1998). An integrated nanoliter DNA analysis device. Science 282, 484-487.

Cristini V and Y. Tan (2004). Theory and numerical simulation of droplet dynamics in complex flows-a review. Lab Chip. 4, 257-264.

Ganan-Calvo A.M. (1998). Generation of Steady Liquid Microthreads and Micron-Sized Monodisperse Sprays in Gas Streams. Phys Rev Lett. 80, 285-288.

Garstecki P., M.J. Fuerstman, H.A. Stone and G. M. Whitesides (2006). Formation of droplets and bubbles in a microfluidic $\mathrm{T}$ junctions- scaling and mechanism of break-up. Lab on a Chip. 6, 437-446.

Gupta R., D.F. Fletcher, and B.S. Haynes (2009). On the CFD modeling of Taylor flow in microchannels. Chemical Engineering Science 64, 2941-2950.

Jullien M.-C., M.-J. Tsang Mui Ching, C. Cohen, L. Menetrier and P. Tabeling (2009). Droplet breakup in microfluidic $\mathrm{T}$-junctions at small capillary numbers, Phys. Fluids 21, 072001.

Karniadakis G., A. Beskok and N. Aluru (2004). Microflows and Nanoflows Fundamentals and Simulation. 2nd ed. Springer, New York, 2005.

Lafaurie B., C. Nardone, R. Scardovelli, S. Zaleski and G. Zanetti (1994), J. Comput. Phys. 113, 134 (1994).

Leshansky A.M., L.M. Pismen (2009). Breakup of drops in a microfluidic T-junction. Physics of Fluids. 21, 023303.

Link D.R., S.L. Anna, D.A.Weitz and H.A. Stone. 2004. Geometrically mediated breakup of drops in microfluidic devices. Phys. Rev. Lett. 92, 054503.

Prakash M. and N. Gershenfeld (2007). Microfluidic bubble logic. Science 315, 832.

Renardy Y.(2007). The effects of confinement and inertia on the production of droplets. Rheol Acta $46,521-529$. 
A. Bedram et al. / JAFM, Vol. 6, No. 1, pp. 81-86, 2013.

Scardovelli R. and Zaleski S. (1999). Direct numerical simulation of free-surface and interfacial flow. Annu. Rev. Fluid Mech. 31,567603.

Sugiura S., M. Nakajima, S. Iwamoto and M. Seki (2001). Langmuir 17, 5562- 5566.

Sybulski O. and P. Garstevki (2010). Dynamic memory in a microfluidic system droplets traveling through a simple network of microchannels. Lab on a chip. 4, 484-493.
Thorsen T., R.W. Roberts, F. H. Arnold, and S. R. Quake (2001). Dynamic Pattern Formation in a Vesicle-Generating Microfluidic Device Phys Rev Lett. 86, 4163-4166.

Tice, J., H. Song and A. Lyon, R. Ismagilov (2003). Formation of droplets and mixing in multiphase microfluidics at low values of the Reynolds and the capillary numbers. Langmuir 19, 912739133.

Urbant P., A Leshansky and Yu Halupovich. (2008). On the forced convective heat transport in a droplet-laden flow in microchannels. Microfluid Nanofluid 4, 533-542. 